\title{
Comparison of the performance of the rapid antigen detection actim Influenza A\&B test and RT-PCR in different respiratory specimens
}

\author{
Correspondence \\ B. Ghebremedhin \\ beniam.ghebremedhin \\ @med.ovgu.de
}

Received 22 June 2008

Accepted 14 November 2008

\author{
B. Ghebremedhin, ${ }^{1}$ I. Engelmann, ${ }^{2}$ W. König ${ }^{1}$ and B. König ${ }^{1}$ \\ ${ }^{1}$ University Clinic Magdeburg, Institute of Medical Microbiology, Magdeburg, Germany \\ ${ }^{2}$ Institute of Virology, Hannover Medical School, Hannover, Germany
}

\begin{abstract}
Nowadays, influenza antigen detection test kits are used most frequently to detect influenza A or B virus to establish the diagnosis of influenza rapidly and initiate appropriate therapy. This study was conducted to evaluate the performance of the actim Influenza A\&B test (Medix Biochemica).

Overall, 473 respiratory specimens were analysed in the actim Influenza A\&B test and the results were compared with those from an RT-PCR assay; 461 of these samples originated from paediatric patients aged 7 weeks to 6.5 years either with influenza-related symptoms or from the intensive care unit, and 12 samples originated from adults with underlying lung or haematological diseases. Diagnosis of influenza A or B virus could be established using the actim Influenza A\&B test (9/473 samples for influenza $A$ virus and $6 / 473$ for influenza $B$ virus). RT-PCR revealed 23 patients with influenza virus (13/473 for influenza $A$ virus and 10/473 for influenza $B$ virus). The sensitivity and specificity of the actim Influenza A\&B test were 65 and $100 \%$ compared with the RT-PCR assay. However, 32 external quality assessment samples containing seven different strains of influenza $A$ subtypes $\mathrm{H} 1 \mathrm{~N} 1$ and $\mathrm{H} 3 \mathrm{~N} 2$ and the avian $\mathrm{H} 5 \mathrm{~N} 1$ were detected correctly by the actim Influenza A\&B test. No cross-reactivity to a range of bacterial, fungal and other viral pathogens was observed. In conclusion, the actim Influenza A\&B test is reliable for positive results due to its high specificity. Nevertheless, negative results from this test need to be confirmed by a more sensitive assay because of the low sensitivity observed with diagnostic samples.
\end{abstract}

\section{INTRODUCTION}

Influenza viruses cause significant morbidity and mortality in both children and adults (Heikkinen, 2006; van der Wouden et al., 2006) during local outbreaks or epidemics. Outbreaks and epidemics may appear at any time but are usually concentrated in the winter months in countries with temperate climates (Brammer, 2002).

The rapid detection of influenza viruses is important to apply preventative strategies, initiate antiviral therapy, detect avian influenza viruses and exclude influenza-like bacterial and viral diseases (e.g. anthrax, smallpox). Moreover, it is important to determine not only whether influenza virus is present, but also which type of influenza virus is present (Noyola et al., 2000a; Chan et al., 2002; Effler et al., 2002; Poehling et al., 2002).

Although the gold standard reference method for the diagnosis of influenza is isolation of the virus, commercially available rapid antigen detection tests have the advantage of providing results much more quickly, i.e. within approximately $30 \mathrm{~min}$. These tests, also referred to as near-patient or point-of-care tests, are mostly immunoassays that detect influenza virus antigen, although one test detects viral neuraminidase activity (Noyola et al., 2000b; Uyeki, 2003).
They detect and distinguish between influenza A and B viruses, detect but do not distinguish between influenza $\mathrm{A}$ and $B$ viruses or detect influenza $A$ virus only. The tests vary in complexity, type of respiratory specimen acceptable for testing and the time needed to obtain results (Seno et al., 1990; Rodriguez et al., 2002; Uyeki, 2003; Hurt et al., 2007).

The actim Influenza $A \& B$ test (Medix Biochemica) is a point-of-care test utilizing influenza-specific $\mathrm{mAbs}$ for rapid diagnosis and differentiation of influenza A and B viruses. The aim of this study was to conduct a prospective evaluation of this test for qualitative, simultaneous and differential detection of influenza $\mathrm{A}$ and $\mathrm{B}$ antigens in different respiratory specimens. For this purpose, the actim Influenza $\mathrm{A} \& \mathrm{~B}$ test was compared with an established inhouse RT-PCR assay (Schweiger et al., 2000) for sensitivity, specificity and the time needed to obtain results.

\section{METHODS}

Viruses and other micro-organisms tested. The performance of the actim Influenza $A \& B$ test was assessed for human influenza $A$ virus $(\mathrm{H} 1 \mathrm{~N} 1, \mathrm{H} 3 \mathrm{~N} 2$ and $\mathrm{H} 5 \mathrm{~N} 1)$ and influenza $\mathrm{B}$ virus samples $(n=32)$ obtained from the quality assurance institution INSTAND 
(Düsseldorf, Germany) (Table 1). A panel of other micro-organisms, including 11 bacteria (Citrobacter freundii, Enterobacter cloacae, Escherichia coli, Haemophilus sp., Klebsiella pneumoniae, Legionella pneumophila, Mycoplasma pneumoniae, Pseudomonas aeruginosa, Staphylococcus aureus, Staphylococcus epidermidis and Streptococcus pneumoniae), four Candida spp. (C. albicans, C. glabrata, C. krusei and $C$. tropicalis) and ten viruses (adenovirus, human bocavirus, human cytomegalovirus, enterovirus, Epstein-Barr virus, herpes simplex virus, human metapneumovirus, respiratory syncytial virus, rhinovirus and varicella-zoster virus) were used to test for crossreactivity in the actim Influenza A\&B test. Bacteria and yeasts were cultured and resuspended in Dulbecco's PBS at a concentration of $10^{7}-10^{8}$ c.f.u. $\mathrm{ml}^{-1}$ or, in the case of $M$. pneumoniae, at a concentration of $10^{7}-10^{8}$ colour-changing units $\mathrm{ml}^{-1}$. For testing cross-reactivity to viruses other than influenza, patient specimens positive for the respective viruses were used. The actim Influenza A\&B test was carried out with the investigator blinded to the nature of the pathogen.

Patients and clinical specimens. Paediatric patients $(n=461)$ who presented (if they presented with symptoms) with fever, cough, coryza and/or myalgia/headache were eligible. Paediatric patients with immunosuppression secondary to drugs and human immunodeficiency virus infection were excluded from enrolment in the study. Nasopharyngeal aspirates and swabs $(n=367)$, bronchoalveolar lavages $(n=11)$ and the remainder as tracheal secretions, submitted for virus diagnostics between November 2004 and April 2005, October 2005 and April 2006, September 2006 and January 2007, and October 2007 and April 2008, were used for this study. The age of the patients ranged from 7 weeks to 6.5 years with the majority $(63 \%)$ being between 0.5 and 1.5 years. Secretions were aspirated into a mucus trap by inserting a catheter into the nasopharynx of each nostril in turn. Specimens were collected at the time of admission and held at $4-8{ }^{\circ} \mathrm{C}$ (for periods of $1-6 \mathrm{~h}$, depending on time of admission) until they were transported to the laboratory. Specimens collected more than $24 \mathrm{~h}$ prior to arrival in the laboratory were excluded from the study. Transport to the laboratory was carried out at ambient temperature; the maximal duration of exposure to ambient temperature was less than $3 \mathrm{~h}$. Aliquots of the respiratory specimens were taken for RT-PCR assay of the respiratory viruses. A second aliquot was used for analysis in the actim Influenza A\&B test and a third aliquot was frozen at $-70{ }^{\circ} \mathrm{C}$ for resolution of discrepant results.

Additionally, 12 respiratory specimens (stored at $-20{ }^{\circ} \mathrm{C}$ ) collected from adults with underlying lung or haematological diseases who had tested positive for influenza virus were included in the panel of 473 samples.

Ethical approval was obtained from the institutional review boards for this study.

actim Influenza A\&B test. The actim Influenza A\&B test was carried out according to the manufacturer's instructions. The test was performed with the investigator blinded to the results of the RT-PCR. For liquid specimens, $300 \mu \mathrm{l}$ sample was dispensed into the test tube together with three drops of extraction buffer, and nasopharyngeal swabs were inserted into test tubes containing six drops of $\mathrm{NaCl}$ buffer and three drops of extraction buffer, mixed and incubated for $5 \mathrm{~min}$ at room temperature to allow virus particles in the specimens to be disrupted, exposing internal viral nucleoproteins. The appearance of a blue-black control line on the actim test strip confirmed that the test had been performed properly and that the results were therefore valid.

BinaxNOW Influenza A \& B test. The BinaxNOW Influenza A \& B test (Binax) is an immunochromatographic membrane assay that uses highly sensitive mAbs to detect influenza type A and B nucleoprotein antigens in nasopharyngeal specimens. The test was carried out according to the manufacturer's instructions.

RNA extraction and cDNA synthesis. Total viral RNA was extracted using a commercial kit (QIAamp Viral RNA kit; Qiagen) according to the manufacturer's instructions. Viral RNA was extracted from $140 \mu \mathrm{l}$ of each sample. The RNA was eluted from columns with $50 \mu \mathrm{l}$ RNase-free water (Qiagen) and used immediately in the following experiments or stored at $-80{ }^{\circ} \mathrm{C}$. cDNA synthesis

Table 1. RT-PCR and actim Influenza $A \& B$ test results for the influenza $A$ and $B$ virus quality assessment strains

NA, Not applicable.

\begin{tabular}{|c|c|c|c|c|c|}
\hline \multirow[t]{2}{*}{ Controls/samples } & \multicolumn{2}{|c|}{ Influenza $A$ virus } & \multicolumn{2}{|c|}{ Influenza $B$ virus } & \multirow[t]{2}{*}{ Virus designation } \\
\hline & RT-PCR & actim & RT-PCR & actim & \\
\hline Control $^{*}$ & NA & + & NA & - & A/Taiwan/1/1986 (H1N1) \\
\hline Control $^{*}$ & NA & - & NA & + & B/Qingdao/102/1991 \\
\hline $20033,20039,20043,20052$ & + & + & - & - & A/New Caledonia/20/1999 (H1N1) \\
\hline $20034,20041,20044$ & - & - & + & + & B/Hong Kong/330/2001 \\
\hline 20036 & + & + & - & - & A/Moscow/10/99 (H3N2) \\
\hline 20038 & + & + & - & - & A/Wyoming/3/2003 (H3N2) \\
\hline 20040, 20042, 20053, 20061 & - & - & + & + & B/Shanghai/361/2002 \\
\hline 20051,20054 & + & + & - & - & A/California/7/04 (H3N2) \\
\hline $20055,20057,20060,20062$ & + & + & - & - & A/Duck/Vietnam/TG24-01/2005 (H5N1) \\
\hline $20066,20070,20073$ & + & + & - & - & A/Wisconsin/67/2005 (H3N2) \\
\hline $20064,20072,20074$ & - & - & + & + & B/Malaysia/2506/2004 \\
\hline $20069,20063,20071,20075$ & + & + & - & - & A/Whooper swan/Germany/R65-2/2006 (H5N1) $\dagger$ \\
\hline $20067,20068,20076$ & + & + & - & - & A/Fukushima/141/2006 (H1N1) \\
\hline
\end{tabular}

${ }^{\star}$ Controls supplied with the actim Influenza A\&B test kit.

$\dagger \mathrm{H} 5 \mathrm{~N} 1$ avian influenza virus strain. 
was carried out at $37^{\circ} \mathrm{C}$ for $1 \mathrm{~h}$ using $10 \mu$ eluted RNA, $100 \mathrm{U}$ murine leukaemia virus reverse transcriptase (Gibco/Invitrogen), $10 \mathrm{mM}$ dithiothreitol, $20 \mathrm{U}$ RNasin (Promega) and $0.25 \mu \mathrm{M}$ random hexamer primers (Gibco/Invitrogen).

FastCycler PCR. PCR was carried out in a $20 \mu$ l volume containing $2.5 \mu \mathrm{l}$ cDNA, $2 \times$ master mix (FastCycler 9800, $2 \times$ GeneAmp Fast PCR Master Mix; Applied Biosystems) and $0.25 \mu \mathrm{M}$ each primer. Primer sets specific for influenza A and B virus (custom synthesized by MWG-Biotech) were used to detect and differentiate the two influenza viruses as described by Schweiger et al. (2000). PCR conditions were initial denaturation at $95{ }^{\circ} \mathrm{C}$ for $10 \mathrm{~s}, 40$ cycles of $95{ }^{\circ} \mathrm{C}$ for $2 \mathrm{~s}$ and annealing and extension at $67{ }^{\circ} \mathrm{C}$ for $15 \mathrm{~s}$, and final extension at $72{ }^{\circ} \mathrm{C}$ for $7 \mathrm{~min}$. Amplicons were detected by gel electrophoresis.

Statistical analysis. Specimens positive for influenza A or B virus in the RT-PCR were regarded as true positives. The sensitivity, specificity, and positive (PPV) and negative (NPV) predictive values of the actim Influenza $\mathrm{A} \& \mathrm{~B}$ test results compared with those of the RT-PCR assay were calculated using two-by-two contingency tables.

\section{RESULTS}

\section{Cross-reactivity of the actim Influenza A\&B test}

To determine the cross-reactivity of the antigen detection kit actim Influenza $\mathrm{A} \& \mathrm{~B}$, commensal and pathogenic micro-organisms (11 bacteria, 10 viruses and four Candida species) that may be present in the nasal cavity or nasopharynx were tested. All of the samples were negative by the actim Influenza $A \& B$ test.

\section{Analytical sensitivity of the actim Influenza A\&B test in quality assessment influenza samples}

A total of 32 quality assessment influenza A and B samples of different designation (INSTAND), including avian influenza virus, were evaluated by the actim Influenza $\mathrm{A} \& \mathrm{~B}$ test and RT-PCR. All of these samples tested positive by both methods (Table 1), revealing a sensitivity and specificity of $100 \%$ for the actim Influenza A\&B test.

\section{Comparison of the actim Influenza A\&B test and RT-PCR assay}

Among the 473 specimens, nine (1.9\%) were antigenpositive for influenza $A$ virus in the actim Influenza $A \& B$ test and $13(2.8 \%)$ were positive in the RT-PCR assay. Six specimens $(1.3 \%)$ were positive for influenza $\mathrm{B}$ virus by the actim Influenza A\&B test and ten (2.1\%) in the RTPCR assay (Table 2). All influenza samples that were detected by the actim Influenza A\&B test were also positive in the RT-PCR assay.

The results for the first batch of PCR amplicons $(n=9)$ were confirmed by sequencing, whilst the other results were confirmed by haemagglutinin and neuraminidase genespecific PCR or by the use of other detection methods (virus culture, direct immunofluorescence, rapid antigen detection
Table 2. Comparison of the actim Influenza A\&B test with RTPCR in detection of influenza $A$ and $B$ viruses in respiratory specimens $(n=473)$

\begin{tabular}{|lcc|}
\hline & \multicolumn{2}{c|}{ RT-PCR } \\
\cline { 2 - 3 } & Positive & Negative \\
\hline Influenza A & & \\
actim-positive & 9 & 0 \\
actim-negative & 4 & 460 \\
Total & 13 & 060 \\
Influenza B & & \\
actim-positive & 6 & 463 \\
actim-negative & 4 & 463 \\
Total & 10 & \\
\hline
\end{tabular}

test or RT-PCR). Of the influenza A viruses detected, 11 were H3N2 and two were H1N1 (data not shown).

The overall sensitivity of the actim Influenza $A \& B$ test compared with the RT-PCR assay was $65.2 \%$ (69.2\% for influenza $\mathrm{A}$ virus and $60 \%$ for influenza $\mathrm{B}$ virus) with a specificity of $100 \%$. The actim Influenza A\&B test revealed a PPV of $100 \%$ (95\% CI 78.2-100\%) for both influenza virus types, whereas the NPV was approximately $98 \%$ (95\% CI 96.5-99.2\%).

From the panel of 473 samples, 149 paediatric respiratory samples were also analysed using a BinaxNOW Influenza A \& B kit. The sensitivity of the BinaxNOW Influenza A \& B kit [4/7 RT-PCR-positive samples (57\%) for influenza A virus and $3 / 7$ RT-PCR-positive samples $(43 \%)$ for influenza $B$ virus] was lower than the sensitivity of the actim Influenza A\&B test $(71 \%$ for influenza A virus and $57 \%$ for influenza B virus when compared for the 149 samples). The BinaxNOW Influenza A \& B test kit revealed a specificity of $100 \%$ for both influenza A and B viruses compared with the RT-PCR assay. When compared with the actim Influenza $\mathrm{A} \& \mathrm{~B}$ test as the reference, the BinaxNOW Influenza A \& B test yielded a sensitivity of $78 \%$. RT-PCR-positive samples were confirmed as described above.

\section{Clinical symptoms of influenza A and B virus- positive cases}

The majority of the influenza virus-positive samples were detected between February and March 2005 and between January and April 2008. Among the patients with influenza, the youngest was 7 weeks old and the eldest 61 years old (with influenza A and B virus, respectively; Table 3). The clinical presentation of the patients with influenza virus detection as well as the type of specimen in which influenza virus was detected are shown in Table 3. No dual infections with influenza A and B viruses were detected. 
Table 3. Characteristics of influenza $A$ and $B$ virus-positive patients and samples

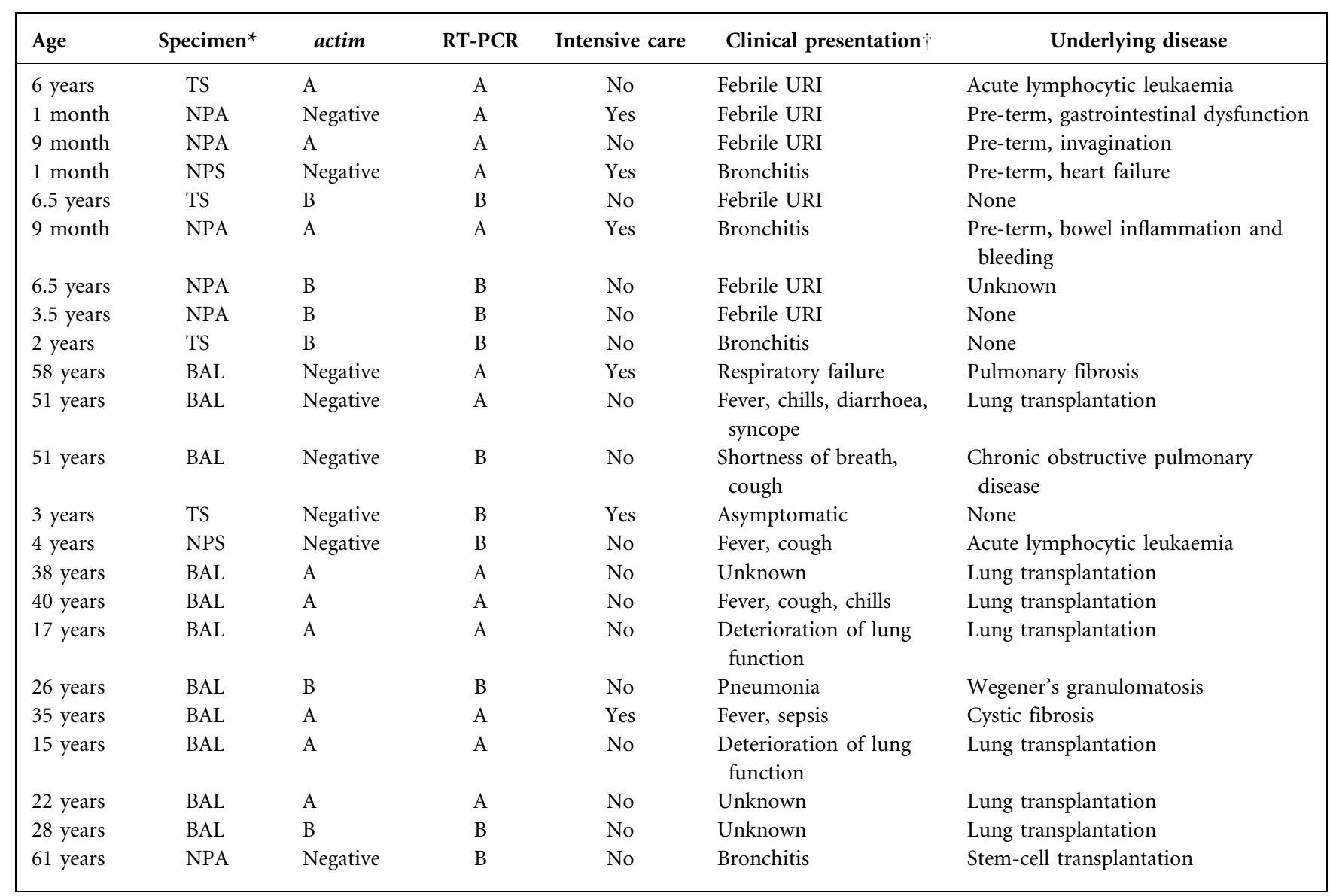

${ }^{\star}$ BAL, Bronchoalveolar lavage; NPA, nasopharyngeal aspirate; NPS, nasopharyngeal swab; TS, tracheal secretion.

$\dagger$ URI, Upper respiratory tract infection.

\section{DISCUSSION}

Although the gold standard for detecting influenza virus infection is virus isolation in cell culture, the result is usually obtained too late to initiate antiviral treatment in time and to take measures to prevent transmission to other patients. Therefore, tests that yield results in a time of minutes to hours can improve the clinical management of suspected influenza cases among outpatients, emergency room patients and inpatients. Currently available tests that produce results in this time frame include immunofluorescence (direct and indirect), RT-PCR, real-time PCR and rapid antigen detection tests. Generally, positive results by the rapid antigen detection methods correlate well with actual infection as determined by shell vial culture (Dunn et al., 2003). The sensitivities of the different rapid assays for influenza virus available at present vary from 40 to $96 \%$ among different studies, depending on the reference methods used (Noyola et al., 2000b; Herrmann et al., 2001; Chan et al., 2002; Effler et al., 2002; Poehling et al., 2002; Dunn et al., 2003; Hurt et al., 2007).

For example, Herrmann et al. (2001) compared an optical immunoassay for rapid detection of influenza $\mathrm{A}$ and $\mathrm{B}$ virus with RT-PCR and immunofluorescence on nasopharyngeal aspirates and swabs. The sensitivity and specificity of the optical immunoassay in nasopharyngeal aspirates were 40.4 and $94.3 \%$, respectively, compared with the RT-PCR. In general, a greater yield of positive samples can be obtained with PCR techniques than with rapid antigen detection tests. This has been observed particularly when the sample is taken 3-4 days after the onset of symptoms, when the viral load drops sharply, as does the infecting capacity (Cherian et al., 1994).

The Directigen FluA $+\mathrm{B}$ antigen test (BD Diagnostic Systems) was evaluated by Chan et al. (2002) in a study design similar to our present work. Most of the patients studied were children. Compared with an RT-PCR assay, the sensitivity, specificity, and PPV and NPV of the Directigen FluA + B test for influenza virus A were 96, 99.6, 96 and $99.6 \%$, and for influenza virus B were 87.5, 96.8, 80 and $98 \%$, respectively. In a French study of the QuickVue Influenza antigen test (Quidel), these values were 87.5, 100, 100 and $89.5 \%$, respectively (Arsene et al., 2004). These results seem to suggest a better performance of the Directigen FluA $+B$ and QuickVue Influenza 
antigen tests than of the actim Influenza $A \& B$ test in the present study. However, Ruest et al. (2003) compared a number of antigen tests with an RT-PCR assay with different results. A poor specificity (35-58 \%) and a poor PPV (41-60\%) were reported for the compared antigen tests, with the Directigen FluA $+B$ test having a higher sensitivity than the QuickVue Influenza antigen test but being associated with a larger number of invalid results. For comparison, the actim Influenza $A \& B$ test had a sensitivity, specificity, and PPV and NPV of 65, 100, 100 and $98 \%$, respectively, and no invalid results were observed in the present study. Cross-reactivity of the actim Influenza $\mathrm{A} \& \mathrm{~B}$ test with other bacteria, fungi and viruses that may be encountered in the respiratory tract was not observed. Moreover, false-positive results were not obtained using the actim Influenza $A \& B$ test in the panel of 473 patient specimens tested (Table 2). Therefore, the specificity and robustness of the actim Influenza A\&B test are probably superior to those of the above-described rapid antigen detection kits.

As the sensitivity of the actim Influenza $A \& B$ assay in diagnostic samples seemed to be lower than that of the above-mentioned antigen detection kits, we analysed a subgroup of 149 respiratory samples in a direct comparison of the BinaxNow Influenza A \& B and actim Influenza A\&B tests. This revealed that the BinaxNow Influenza A \& B test did not yield better results than the actim Influenza $A \& B$ test. Moreover, the actim Influenza A\&B assay achieved a sensitivity of $100 \%$ in comparison with RT-PCR and the expected results in a panel of quality assessment samples (including influenza A H1N1, H3N2 and H5N1) obtained from INSTAND (Table 1).

In summary, the studies cited above, together with our results, suggest that the reported sensitivities of influenza rapid antigen tests may vary highly in relation to the panel of diagnostic specimens tested. If a panel contains diagnostic specimens with low virus concentrations (perhaps because these were taken more than 2 days after the onset of symptoms), the sensitivities of antigen assays drop in comparison with that of RT-PCR, which can still detect influenza RNA due to its low detection limit. Thus RT-PCR offers an alternative method of diagnosing influenza virus infections. It has high sensitivity and specificity (Herrmann et al., 2001), but requires a high level of skill and a complex laboratory infrastructure, and takes several hours to perform, as was also shown in our study (150-170 min). Therefore, RT-PCR does not fill the niche of tests that are rapid and easy to perform with a low level of expertise.

The low influenza activity in the Magdeburg area during the respective seasons between 2004 and 2008 was disadvantageous for this study, resulting in a limited number of positive specimens being obtained. Thus studying a larger number of positive specimens would make evaluation and determination of sensitivity and specificity more reliable. However, during times of low influenza incidence, the NPV of a rapid antigen detection test is higher and the PPV is lower than in times of high influenza activity (WHO, 2005). Therefore, the high PPV of the actim assay observed in this study indicates that positive results do not need to be confirmed by RT-PCR and should be reported to the treating physician immediately in times of both low and high influenza incidence. However, negative actim Influenza A\&B results, as well as negative results of other rapid antigen assays, should be confirmed by RT-PCR. This is even more important during the influenza season, because of decreasing NPV with increasing influenza virus incidence.

In conclusion, the actim Influenza $\mathrm{A} \& \mathrm{~B}$ test is a rapid in vitro assay that detects and differentiates influenza $\mathrm{A}$ and $\mathrm{B}$ virus antigens (nucleoprotein) extracted from respiratory specimens. Because the actim Influenza A\&B test is easy to perform and has a specificity and PPV of $100 \%$ for each virus, this test is appropriate for acute cases as a first-line emergency diagnostic method. A negative result warrants confirmation with a more sensitive method.

\section{ACKNOWLEDGEMENTS}

Part of this study was presented during the 107th ASM General Meeting in Toronto, Canada, May 2007. No financial support was obtained for this study. The authors have no potential conflicts of interest.

\section{REFERENCES}

Arsene, S., Vabret, A., Dina, J., Tecu, C., Brouard, J., Eckard, P., Margry, P., Joannes, M. \& Freymuth, F. (2004). Comparison of the Quick Vue Influenza test (Quidel) to an immunofluorescence assay for the detection of influenza virus infections. Roum Arch Microbiol Immunol 63, 235-243.

Brammer, T. L., Murray, E. L., Fukuda, K., Hall, H. E., Klimov, A. \& Cox, N. J. (2002). Surveillance for influenza - United States 1997-98, 1998-99, and 1999-00 season. MMWR Surveill Summ 51, 1-10.

Chan, K. H., Maldeis, N., Pope, W., Yup, A., Ozinskas, A., Gill, J., Seto, W. H., Shortridge, K. F. \& Peiris, J. S. (2002). Evaluation of the Directigen Flu A + B test for rapid diagnosis of influenza virus type A and B infections. J Clin Microbiol 40, 1675-1680.

Cherian, T., Bobo, L., Steinhoff, M. C., Karron, R. A. \& Yolken, R. H. (1994). Use of PCR-enzyme immunoassay for identification of influenza A virus matrix RNA in clinical samples negative for cultivable virus. J Clin Microbiol 32, 623-628.

Dunn, J. J., Gordon, C., Kelley, C. \& Carroll, K. C. (2003). Comparison of the Denka-Seiken INFLU A.B-Quick and BD Directigen Flu A+B kits with direct fluorescent-antibody staining and shell vial culture methods for rapid detection of influenza viruses. J Clin Microbiol 41, 2180-2183.

Effler, P. V., leong, M. C., Tom, T. \& Nakata, M. (2002). Enhancing public health surveillance for influenza virus by incorporating newly available rapid diagnostic tests. Emerg Infect Dis 8, 23-28.

Heikkinen, T. (2006). Influenza in children. Acta Paediatr 95, 778784.

Herrmann, B., Larsson, C. \& Zweygberg, B. W. (2001). Simultaneous detection and typing of influenza viruses $A$ and $B$ by a nested reverse transcription-PCR: comparison to virus isolation and antigen 
detection by immunofluorescence and optical immunoassay (FLU OIA). J Clin Microbiol 39, 134-138.

Hurt, A. C., Alexander, R., Hibbert, J., Deed, N. \& Barr, I. G. (2007). Performance of six influenza rapid tests in detecting human influenza in clinical specimens. J Clin Virol 39, 132-135.

Noyola, D. E., Clark, B., O’Donnell, F. T., Atmar, R. L., Greer, J. \& Demmler, G. J. (2000a). Comparison of a new neuraminidase detection assay with an enzyme immunoassay, immunofluorescence, and culture for rapid detection of influenza A and B viruses in nasal wash specimens. J Clin Microbiol 38, 1161-1165.

Noyola, D. E., Paredes, A. J., Clark, B. \& Demmler, G. J. (2000b). Evaluation of a neuraminidase detection assay for the rapid detection of influenza A and B virus in children. Pediatr Dev Pathol 3, 162-167.

Poehling, K. A., Griffin, M. R., Dittus, R. S., Tang, Y. W., Holland, K., Li, H. \& Edwards, K. M. (2002). Bedside diagnosis of influenza virus infections in hospitalized children. Pediatrics 110, 83-88.

Rodriguez, W. J., Schwartz, R. H. \& Thorne, M. M. (2002). Evaluation of diagnostic tests for influenza in a pediatric practice. Pediatr Infect Dis J 21, 193-196.
Ruest, A., Michaud, S., Deslandes, S. \& Frost, E. H. (2003). Comparison of the Directigen Flu A + B test, the QuickVue influenza test, and clinical case definition to viral culture and reverse transcription-PCR for rapid diagnosis of influenza virus infection. $J$ Clin Microbiol 41, 3487-3493.

Schweiger, B., Zadow, I., Heckler, R., Timm, H. \& Pauli, G. (2000). Application of a fluorogenic PCR assay for typing and subtyping of influenza viruses in respiratory samples. J Clin Microbiol 38, 15521558.

Seno, M., Kanamoto, Y., Takao, S., Takei, N., Fukuda, S. \& Umisa, H. (1990). Enhancing effect of centrifugation on isolation of influenza virus from clinical specimens. J Clin Microbiol 28, 16691670 .

Uyeki, T. M. (2003). Influenza diagnosis and treatment in children: a review of studies on clinically useful tests and antiviral treatment for influenza. Pediatr Infect Dis J 22, 164-177.

van der Wouden, J. C., Bueving, H. J. \& Thomas, S. (2006). Influenzaassociated deaths among children. N Engl J Med 354, 1317-1318.

WHO (2005). Recommendations on the Use of Rapid Testing for Influenza Diagnosis. Geneva: World Health Organization. 